\title{
Design of a Non-Overshooting PID Controller with an Integral Sliding Perturbation Observer for Motor Positioning Systems*
}

\author{
Yu-Sheng LU** and Chao-Min CHENG**
}

\begin{abstract}
This paper describes the design of a non-overshooting proportional-integral-derivative (PID) controller with both pre-assigned initial integral state and a perturbation compensation scheme for motor positioning systems. According to the requirement of non-overshooting output response and the specified settling time in tracking a step reference signal, the PID gains and the initial integral state are determined systematically. The proposed PID control provides a continuous control signal when the nominal system is subject to sudden set-point changes, implying smooth system performance. To compensate for the system perturbation resulting from parameter variations and unknown load disturbances, an integral sliding perturbation observer is proposed and integrated into the PID-controlled system, which requires neither access to the time derivative of the state vector nor accurate knowledge of system parameters. A sliding mode is ensured throughout the entire response, and system perturbation is estimated by feeding a switching signal through an integrator. Due to the low-pass filtering characteristic of the integral action and the reduction of the required switching gain, the proposed scheme not only precisely compensates for system perturbation within the interested frequency range, but also effectively alleviates the chattering problem in control signals. The usefulness of the proposed design is demonstrated through an experimental study of a motor drive under load variations and disturbances.
\end{abstract}

Key Words: PID Control, Non-Overshooting Servo-Control, Sliding Mode, Perturbation Observer

\section{Introduction}

In practical motor positioning systems, an inner feedback loop of current control possessing fast dynamics is employed with the ease of current limitation, while an outer feedback loop of position control generates current commands to the inner-loop current controller. The proportional-integral-derivative (PID) control scheme has gained widespread use in the position control of such motor drives. Many methods for determining the PID gains have been proposed and surveyed in Refs. (1) - (3). Apart from its simple structure and its straightforward microprocessor implementation, the PID controller provides the ability of maintaining zero steady-state error to a step reference change by using the integral action. Generally

* Received 7th April, 2004 (No. 04-5047)

** Department of Mechanical Engineering, National Yunlin University of Science and Technology, 123 University Road, Section 3, Touliu, Yunlin 640, Taiwan.

E-mail: luys@yuntech.edu.tw speaking, in addition to yielding zero steady-state error, high-performance motor positioning systems should track set-point changes rapidly without overshoot and be robust to parameter variations and load disturbances. However, with a fixed-gain PID controller having the commonlyused one-degree-of-freedom (1DOF) structure, the maximum overshoot and the speed of response conflict with each other. In other words, both small maximum overshoot and fast response speed cannot be achieved simultaneously. By properly tuning the 1DOF PID controller, it is possible to achieve relatively smaller or no overshoot in the transient response to a step reference input. The speed of response, however, then becomes much slower ${ }^{(4)}$, which in general corresponds to a small bandwidth and poor load disturbance attenuation. On the other hand, the Ziegler-Nichols method of parameter tuning, the mostlyemployed tuning method in industry settings, is known to provide good load disturbance attenuation but yield large overshoot.

To reduce the excessive overshoot, involved by the 
Ziegler-Nichols method, the set-point weighting technique $^{(5),(6)}$ is introduced. This method effectively gives a two-degrees-of-freedom (2DOF) system, where the setpoint value for the proportional action is multiplied by a set-point weighting factor. In Ref. (7), the set-point value for each control action is weighted by a corresponding setpoint weighting factor. The drawback of using set-point weightings is an increase in the rise time since the effectiveness of the proportional action is diminished to some extent. Another $2 \mathrm{DOF}$ design ${ }^{(8),(9)}$ utilized the structure of model-following control for variable-speed motor drives, where a reference model is introduced outside the feedback loop in the feedforward path. The feedback characteristics and the characteristics between the reference input and system output can then be adjusted independently. The introduction of the reference model, however, increases the complexity in realizing this control scheme. Moreover, the design ${ }^{(8)}$ invariably results in an overdamped system in the nominal case, leading to a sluggish output response. Other approaches are the fuzzy gain adaptation schemes compared in Ref.(10), where controller parameters are adjusted on-line for good set-point tracking and load disturbance attenuation. A fuzzy-logicbased tuning of set-point weighting is also presented in Ref. (11). However, these approaches do not clearly show how to set the parameters of fuzzy inference systems in a systematic manner, and the complexity of the resulting control systems may increase in such a way that they are not worth implementing in practical situations.

Disturbance observers are useful tools to compensate for unknown load disturbances and are widely adopted in motor control systems. Distinct from the high-gain feedback control, the compensator based on a disturbance observer estimates the unknown load disturbance, and generates just minimal control to govern system dynamics to a nominal one. There are several kinds of disturbance observers, among which the sliding-mode disturbance observer ${ }^{(12)-(18)}$ is outstanding. Due to the inherent invariance property of a sliding mode, a sliding-mode state observer possesses design simplicity and favorable robustness against modeling errors and disturbances. It is thus natural to apply the structure of a sliding-mode state observer to designing a perturbation observer such that the estimation process is robust to modeling errors. The estimate of disturbance by the observer ${ }^{(12)}$ contains switching components which, when introduced to disturbance compensation, may excite unmodeled dynamics and lead to oscillations in the state vector at finite frequency. These oscillations, normally referred to as chatter, are known to result in low-control accuracy, high heat loss in electric power circuit, and high wear of moving mechanical parts $^{(16)}$. A smooth saturation-type function is adopted in Refs. (13) and (14) to approximate the discontinuous function, so as to alleviate the control chatter at the sacri- fice of estimation preciseness. Moreover, the analysis ${ }^{(14)}$ assumed that the time variation of disturbance has negligible effect. In Refs. (15) - (18), the disturbance is shown to be equal to the equivalent value of some switching signal, and its estimate is obtained by feeding the switching signal through a low-pass filter. However, switching gains in these designs must be greater than the bounds on system perturbation. Large switching gains imply significant chatter.

For motor positioning systems, this paper proposed a simple PID control scheme with the initial value of the integral state variable as a design parameter. Thus there are four parameters to be determined in the proposed PID controller: the proportional, integral and derivative gains and the integrator's initial state. According to the desired rise time and the requirement of zero overshoot in the step tracking response, these controller parameters are determined systematically. To enhance the performance robustness of the PID-controlled system, a sliding perturbation observer is proposed and introduced into the control loop with the effect of parameter variations and the external disturbance lumped together as a perturbation term. While the PID-controlled dynamics is tailored to achieve certain performance requirements, the dynamics of perturbation compensation can be tuned fast independently of the tuning of the PID controller. Moreover, the conventionally assumed upper-bounds restriction on the perturbation is relaxed to the restriction on its estimation error, thus requiring a much smaller switching gain for ensuring the existence of a sliding mode. Through this reduction of a switching gain together with the low-pass filtering characteristic of the proposed integral perturbation estimation, the chatter problem is alleviated while system perturbation is precisely compensated for within the frequency range of interest.

\section{Design of a Non-Overshooting PID Controller}

\subsection{Nominal model and performance specifica- tions}

Provided that the current control loop possesses much faster dynamics than the position control loop, the dynamics of the current loop can be neglected in designing a position controller, and the motor-driven system with a single input and a single output is described as a second-order system given by

$$
\ddot{x}+a_{1} \dot{x}+a_{0} x=b(u+d)
$$

in which the coefficients $a_{1}, a_{0}$ and $b$ are the nominal system parameters, $x$ is the motor position, $d$ is the nominal input disturbance, and $u$ is the plant input, namely, the torque-producing current command. Without loss of generality, parameter $b$ is assumed to be positive. In designing a non-overshooting PID controller, it is assumed that the input disturbance $d$ and system parameters $a_{1}, a_{0}$ and $b$ in the nominal model (1) are known in advance. 
For a step reference command $r$, the proposed PID control law is described by

$$
u=u_{p i d}=-K_{D} \dot{x}+K_{P} e+K_{I} z
$$

in which $K_{D}, K_{P}$ and $K_{I}$ denote the derivative, proportional and integral gains, respectively, $e=r-x$, and $z$ denotes an integral state variable given as

$$
z=\int_{0}^{t} e(\tau) d \tau+z_{0}
$$

where $z_{0}$ denotes the initial value of the integral state variable. Note that there are four design parameters: $K_{D}, K_{P}$, $K_{I}$ and $z_{0}$. According to the general requirements of servo drives, the following performance specifications are established: (1) the steady-state error of step tracking response is zero, (2) the overshoot of step transient response is zero, and (3) the settling time is set as $t_{s}$, which is defined as the time required for the step response to reach and stay within $2 \%$ of its final value.

\subsection{Determination of PID gains}

To satisfy the performance specifications and avoid sluggish response, the desired transfer function of the output response $X(s)$ to the reference input $R(s)$ is prescribed as a system with triple poles described by

$$
\frac{X(s)}{R(s)}=\frac{\omega_{n}^{3}}{s^{3}+3 \omega_{n} s^{2}+3 \omega_{n}^{2} s+\omega_{n}^{3}}
$$

in which the undamped natural frequency $\omega_{n}$ can be determined according to the desired settling time $t_{s}$. For a step reference input $r, R(s)=r / s$ and $X(s)$ can be written as

$$
X(s)=\omega_{n}^{3} r /\left(s^{3}+3 \omega_{n} s^{2}+3 \omega_{n}^{2} s+\omega_{n}^{3}\right) s
$$

The inverse Laplace transform of Eq. (5) may be represented by

$$
x(t)=\left\{1-\left(1+\omega_{n} t+\omega_{n}^{2} t^{2} / 2\right) \exp \left(-\omega_{n} t\right)\right\} r \quad \text { for } t \geq 0
$$

The constraint on the settling time $t_{s}$ requires that

$$
x\left(t_{s}\right)=0.98 r=\left\{1-\left(1+\omega_{n} t_{s}+\omega_{n}^{2} t_{s}^{2} / 2\right) \exp \left(-\omega_{n} t_{s}\right)\right\} r
$$

from which the undamped natural frequency is determined as

$$
\omega_{n}=7.5166 / t_{s}
$$

Substituting the control law (2) into system model (1) and noting that $\dddot{z}(t)=-\ddot{x}(t), \ddot{z}(t)=\dot{e}(t)=-\dot{x}(t)$ and $\dot{z}(t)=e(t)=$ $r-x(t)$, we have after rearrangement

$$
\dddot{z}+\left(a_{1}+b K_{D}\right) \ddot{z}+\left(a_{0}+b K_{P}\right) \dot{z}+b K_{I} z=a_{0} r-b d
$$

subject to $\ddot{z}(0)=-\dot{x}_{0}, \dot{z}(0)=e_{0}$ and $z(0)=z_{0}$, where $\dot{x}_{0}$ denotes $\dot{x}(0)$, and $e_{0}$ denotes $e(0)$ and is equal to $r-x(0)$. The characteristic equation for the closed-loop system is thus described by

$$
s^{3}+\left(a_{1}+b K_{D}\right) s^{2}+\left(a_{0}+b K_{P}\right) s+b K_{I}=0
$$

This when compared with the characteristic equation of desired system dynamics (4)

$$
s^{3}+3 \omega_{n} s^{2}+3 \omega_{n}^{2} s+\omega_{n}^{3}=0
$$

yields

$$
K_{D}=\left(3 \omega_{n}-a_{1}\right) / b, \quad K_{P}=\left(3 \omega_{n}^{2}-a_{0}\right) / b, \quad K_{I}=\omega_{n}^{3} / b
$$

For a specified settling time $t_{s}$, the PID gains are determined by Eq. (12), in which the undamped natural frequency is obtained from Eq. (8).

\subsection{Initial setting of the integral state}

The previous pole-placement design yields triple poles on the negative real axis in the complex plane. This, however, does not imply that the integral state variable or the output response would exhibit zero overshoot since the solution of Eq. (9), describing the closed-loop response, depends also on the initial conditions. With the PID gains specified by Eq. (12), let us rewrite Eq. (9) as

$$
\dddot{z}+3 \omega_{n} \ddot{z}+3 \omega_{n}^{2} \dot{z}+\omega_{n}^{3} z=a_{0} r-b d
$$

subject to $\ddot{z}(0)=-\dot{x}_{0}, \dot{z}(0)=e_{0}$ and $z(0)=z_{0}$. Let the initial integral state be of the following form

$$
z_{0}=K_{0} e_{0}+\left(a_{0} r-b d\right) / \omega_{n}^{3}
$$

in which $K_{0}$ is a constant to be determined. From Eqs. (13) and (14), the time solution for the integral state variable can be obtained, from which we have the position response by utilizing the relationship $x(t)=r-\dot{z}(t)$ :

$$
\begin{aligned}
x= & r-\left\{e_{0}-\left(\dot{x}_{0}-e_{0} \omega_{n}\right) t\right. \\
& \left.+\left(\dot{x}_{0} \omega_{n}-2 e_{0} \omega_{n}^{2}-K_{0} e_{0} \omega_{n}^{3}\right) t^{2} / 2\right\} \exp \left(-\omega_{n} t\right)
\end{aligned}
$$

To compare with the desired output response (6), consider the case when $\dot{x}(0)=0$ and $x(0)=0$. Since $\dot{x}_{0}=0$ and $e_{0}=r$ for systems initially at rest, Eq. (15) gives

$$
x=\left\{1-\left[1+\omega_{n} t-\left(2 \omega_{n}^{2}+K_{0} \omega_{n}^{3}\right) t^{2} / 2\right] \exp \left(-\omega_{n} t\right)\right\} r
$$

Equating Eqs. (6) and (16) yields

$$
\omega_{n}^{2}=-\left(2 \omega_{n}^{2}+K_{0} \omega_{n}^{3}\right)
$$

from which we have

$$
K_{0}=-3 / \omega_{n}
$$

Furthermore, according to Eq. (14), the integral state variable is initially assigned as

$$
z_{0}=-3 e_{0} / \omega_{n}+\left(a_{0} r-b d\right) / \omega_{n}^{3}
$$

On the contrary, the integral state variable is always initially set to zero in the conventional PID controller. As the integral state accumulates the position error during the transient response, the closed-loop system tends to exhibit an overshooting output response even when all system poles are located on the negative real axis in the left-half plane. Introducing the flexibility of assigning the initial integral state as given by Eq. (19) to the PID controller avoids this overshoot under the requirement regarding the settling time. 
Remark 1 (Continuity of the control effort): In the conventional PID-controlled system, set-point changes would cause sudden changes in the actuating signal, that is normally undesirable. Suppose that the closed-loop system is in its steady state with $r=r_{0}$ and subject to a step change in the reference signal, say $r=r_{1}$ at $t=t_{1}$. The control input at $t=t_{1}^{-}$can be found as

$$
u\left(t_{1}^{-}\right)=\left(a_{0} r_{0}-b d\right) / b
$$

The control input at $t=t_{1}^{+}$with the proposed PID control is

$$
\begin{aligned}
& u\left(t_{1}^{+}\right)=K_{P}\left(r_{1}-r_{0}\right) \\
& \quad+K_{I}\left[-3\left(r_{1}-r_{0}\right) / \omega_{n}+\left(a_{0} r_{1}-b d\right) / \omega_{n}^{3}\right]
\end{aligned}
$$

With the PID gains determined by Eq. (12), it gives

$$
\begin{aligned}
u\left(t_{1}^{+}\right) & =\left[\left(3 \omega_{n}^{2}-a_{0}\right) / b\right]\left(r_{1}-r_{0}\right) \\
& +\left(\omega_{n}^{3} / b\right)\left[-3\left(r_{1}-r_{0}\right) / \omega_{n}+\left(a_{0} r_{1}-b d\right) / \omega_{n}^{3}\right] \\
& =\left(a_{0} r_{0}-b d\right) / b
\end{aligned}
$$

Comparing Eq. (20) with Eq. (22) yields $u\left(t_{1}^{-}\right)=u\left(t_{1}^{+}\right)$, which means that, when there are abrupt changes in the set-point command, the proposed PID control scheme gives a continuous actuating signal. This also implies a smooth response at the start-up of the system operation.

Remark 2 (Summary of designing the proposed PID controller): Given the performance specifications, the proposed PID control (2) can be designed orderly. According to the desired settling time, the PID gains are obtained by Eqs. (8) and (12). To avoid an overshooting response, the initial integral state is determined by Eq. (19). The design procedure is more systematic than that of the set-point weighting method ${ }^{(5),(6)}$ for motor positioning systems, and introducing the flexibility of assigning the initial integral state does not increase the expected rise time. Moreover, the proposed scheme does not require the implementation of a reference model as necessary in Refs. (8) and (9), thus yielding a simpler controller structure.

\section{Compensation by an Integral Sliding Perturbation Observer}

Instead of attenuating the disturbance effect through high feedback gains, an additional component is incorporated into the control to rectify the perturbation in the PID-controlled system. Consider the following proposed control law in an additive form:

$$
u=u_{p i d}+u_{p c}
$$

in which $u_{p c}$ is the perturbation compensation component defined later.

\subsection{Realistic model and system perturbation}

The practical system model including modeling uncertainties is described by

$$
\ddot{x}+a_{1} \dot{x}+a_{0} x=b(u+\tilde{d})
$$

where $\tilde{d}(t)$ is a lumped term consisting of parameter variations and unknown external disturbance. In the nominal system model (1), the nominal disturbance $d$ is regarded as an estimate of actual disturbance $\tilde{d}(t)$. Substituting Eq. (23) into Eq. (24) gives

$$
\ddot{e}+\left(a_{1}+b K_{D}\right) \dot{e}+\left(a_{0}+b K_{P}\right) e+b K_{I} z=a_{0} r-b \tilde{d}-b u_{p c}
$$

Based on Eqs. (12) and (25), we have

$$
\ddot{e}=-3 \omega_{n} \dot{e}-3 \omega_{n}^{2} e-\omega_{n}^{3} z+a_{0} r-b d+b\left(\psi-u_{p c}\right)
$$

where $\psi=d-\tilde{d}$ and is referred to as system perturbation. Ideally, the component $u_{p c}$ should be set to $\psi$ so that the system perturbation is canceled out from Eq. (26) and the resulting system exhibits the desired output response (6). As the perturbation cannot be measured directly, its estimate generated by the proposed observer is applied to building up this perturbation-compensation component.

\subsection{A sliding mode throughout an entire response}

For the estimation of system perturbation, a sliding mode, which exists during an entire response, is artificially introduced into the following auxiliary process:

$$
\dot{q}=-3 \omega_{n} \dot{e}-3 \omega_{n}^{2} e-\omega_{n}^{3} z+a_{0} r-b d+b \Psi \operatorname{sgn}(\sigma)
$$

in which $q$ is the state variable of the auxiliary process, the switching function $\sigma$ is defined as

$$
\sigma=\dot{e}-q
$$

and the switching gain $\Psi$ is assigned so that $\left|\psi-u_{p c}\right|<\Psi$. Here, $\operatorname{sgn}(\cdot)$ denotes the sign function. To ensure a sliding regime $\sigma=0$, the sliding condition $\lim _{\sigma \rightarrow 0} \sigma \dot{\sigma}<0$ should be satisfied. Taking the derivative of Eq. (28) and substituting Eqs. (26) and (27) into the resulting equation gives

$$
\dot{\sigma}=b\left(\psi-u_{p c}-\Psi \operatorname{sgn}(\sigma)\right)
$$

Multiplying both sides of Eq. (29) by $\sigma$ and noting that $\left|\psi-u_{p c}\right|<\Psi$, we have

$$
\sigma \dot{\sigma}=b\left[\left(\psi-u_{p c}\right) \sigma-\Psi|\sigma|\right]<0, \quad \text { if } \sigma \neq 0
$$

which implies the satisfaction of the sliding condition and the existence of the sliding regime $\sigma=0$ after some time. Subsequently, assigning the initial state of the auxiliary process as $q(0)=\dot{e}(0)$ gives $\sigma=0$ at $t=0$. This together with the satisfaction of the sliding condition implies that

$$
\sigma=0 \text { for all } t \geq 0
$$

Thus, the sliding regime $\sigma=0$ exists for the perturbation estimation during an entire response despite the presence of system perturbation which is desired to be estimated.

\subsection{Integral perturbation compensation}

Since $\sigma=0$ is ensured for all time, we have from Eq. (29)

$$
\dot{\sigma}=b\left(\psi-u_{p c}-\Psi \operatorname{sgn}(\sigma)\right)=0
$$

which leads to

$$
\Psi \operatorname{sgn}(\sigma)=\psi-u_{p c}
$$


in the sense of their equivalent values. Subsequently, the perturbation compensation component $u_{p c}$ is determined through the following integral adaptation law:

$$
\dot{u}_{p c}=k_{C}(t) *[\Psi \operatorname{sgn}(\sigma(t))]
$$

in which $*$ denotes the convolution, and $k_{C}(t)$ is the adaptation gain with its Laplace transform denoted as $k_{C}(s)$. Combining Eqs. (33) and (34) gives

$$
\dot{u}_{p c}=k_{C}(t) *\left(\psi-u_{p c}\right)
$$

and thus

$$
u_{p c}(s)=\frac{k_{C}(s)}{s+k_{C}(s)} \psi(s)
$$

This relationship is invariant to variations of system parameters, and the perturbation compensation component $u_{p c}$ is intrinsically a low-pass-filtered version of system perturbation $\psi$ with the filter's bandwidth determined by the adaptation gain $k_{C}$. Thus, the characteristics of the proposed perturbation observer depend upon the choice of the adaptation gain $k_{C}(s)$. In the absence of any other consideration, a Butterworth configuration is often suitable for designing the low-pass filter. In determining $k_{C}(s)$, let the transfer function from $\psi$ to $u_{p c}$ have a Butterworth polynomial for its denominator. We name the perturbation observer by the order of the corresponding transfer function from $\psi$ to $u_{p c}$.

$$
\begin{array}{ll}
\text { Type I: } & k_{C}(s)=\omega_{0}, \quad \frac{u_{p c}(s)}{\psi(s)}=\frac{\omega_{0}}{s+\omega_{0}} \\
\text { Type II: } & k_{C}(s)=\frac{\omega_{0}^{2}}{s+\sqrt{2} \omega_{0}}, \\
& \frac{u_{p c}(s)}{\psi(s)}=\frac{\omega_{0}^{2}}{s^{2}+\sqrt{2} \omega_{0} s+\omega_{0}^{2}}
\end{array}
$$

in which $\omega_{0}$ determines the cutoff frequency of the transfer function from $\psi$ to $u_{p c}$. Ideally, $u_{p c}$ should be set to be equal to $\psi$. Increasing the value of $\omega_{0}$ approaches this ideal case, improves the preciseness of perturbation compensation, but may increase the chatter level in the control. On the other hand, with the same cutoff frequency $\omega_{0}$, a high-order perturbation observer filters out highfrequency signals more efficiently than a low-order one, but requires more efforts to implement it.

Figure 1 depicts the overall structure of the proposed PID control with perturbation compensation. In the proposed compensation scheme, there are two mechanisms for alleviating the chatter phenomenon in the control. One is the low-pass characteristic of the integral action in Eq. (34) for determining the perturbation compensation component. The other is that the magnitude of the switching gain $\Psi$ can be smaller than that of system perturbation $\psi$. In the previous analysis, the constraint $\Psi>\left|\psi-u_{p c}\right|$ is required to maintain a sliding motion, meaning that the switching gain $\Psi$ needs only to be large enough to cover the range of compensation error $\left(\psi-u_{p c}\right)$. From Eq. (36), it is found that

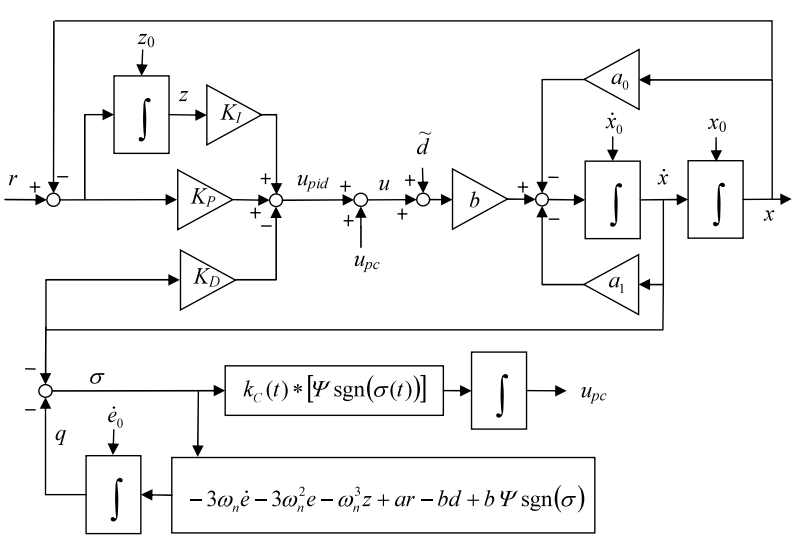

Fig. 1 Block diagram of the proposed PID controller with perturbation compensation

$$
\psi(s)-u_{p c}(s)=\frac{s}{s+k_{C}} \psi(s)
$$

which implies that the system perturbation $\psi$ is effectively attenuated in the low-frequency range, and that the magnitude of compensation error $\left(\psi-u_{p c}\right)$ is smaller than that of $\psi$ itself within the frequency range of interest. The switching gain can thus be chosen smaller than the bounds on system perturbation, alleviating the chatter phenomenon in the actuating signal further. Therefore, the proposed scheme can not only precisely compensate for the system perturbation within the interested frequency range, but also reduce the adverse effect of chattering in the control.

\section{Experimental Study and Validation}

\subsection{System description}

To show the ingredient of the proposed scheme, consider the position control of a permanent-magnet ac servomotor. The experimental system is shown in Fig. 2 with a photo of the electromechanical portion, in which the load consists of an inertia load and another permanent-magnet ac machine, model HA-FE33 manufactured by Mitsubishi Electric, with its three phases connected through $40-\Omega$ resistors in the wye configuration. The ac servomotor driven by a regulated current converter is of model HCKFS73 also from Mitsubishi Electric with a rated torque of $2.4 \mathrm{Nm}$ and rated output power of $750 \mathrm{~W}$. The shaft encoder mounted to the ac servomotor has a resolution of 8000 lines, which yields a resolution of 32000 pulses/rev after the $A$ and $B$ signals from the encoder have been processed by the FPGA (Field Programmable Gate Array), model XCV50PQ240-C6 from Xilinx, Inc.

The velocity information is measured by a digital tachometer that measures the time interval of the encoder pulses to achieve more accurate estimation than the direct differentiation of the position signal. In our experimental system, the tasks of both position counting and velocity detection are implemented in the FPGA. The controller core is a floating-point TMS320C6711 digital signal processor (DSP) which obtains the position and ve- 


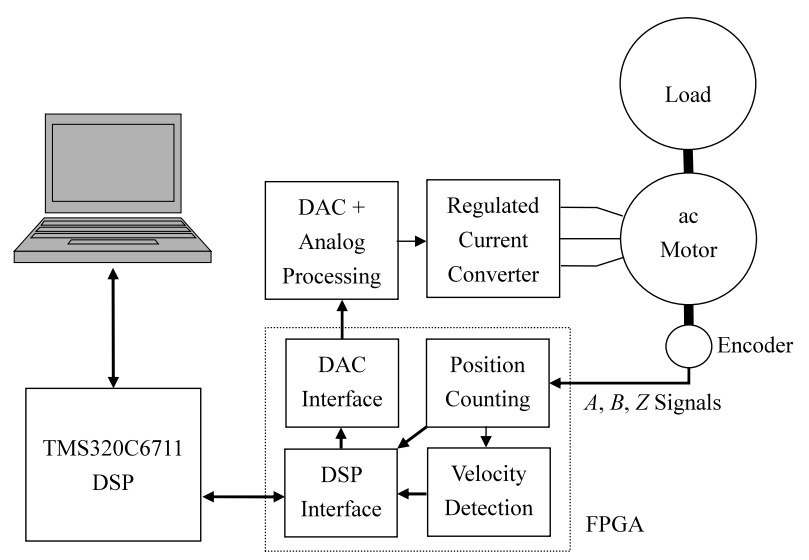

(a)

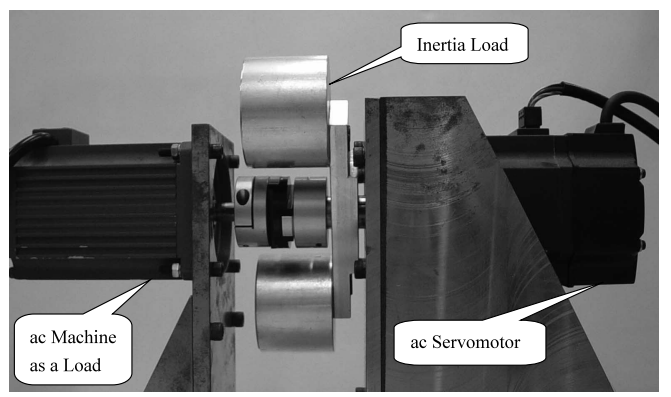

(b)

Fig. 2 Experimental system. (a) Schematic representation of the hardware configuration. (b) Photo of the electromechanical system

locity information from the FPGA, calculates the control algorithms, and sends the control efforts to the regulated current converter through a 12-bit digital-to-analog converter and some analog signal processing circuits. A sampling period of $0.1016 \mathrm{~ms}$ was chosen. In the experimental system, a personal computer was used to develop the control program written in $\mathrm{C}$ language, to compile it, to download the resulting code into DSP for execution and to acquire experimental data.

\subsection{System parameters, performance specifica- tion, and controller design}

Consider Eq. (24) as the mathematical model of our motor drive with $a_{1}=B / J, a_{0}=0$, and $b=K_{t} K_{v 2 c} / J$, in which $B$ is the total viscous-friction coefficient of the motor and load, $J$ is the total inertia constant of the motor and load, $K_{t}$ is the torque parameter of the motor, and $K_{v 2 c}$ is the current gain of the power amplifier. The values of those physical parameters are identified to be $B=3.3432 \mathrm{E}-3(\mathrm{Nm} \cdot \mathrm{s} / \mathrm{rad}), K_{t} K_{v 2 c}=8.3425(\mathrm{Nm} / \mathrm{V})$, and $3.32 \mathrm{E}-4 \leq J \leq 3.15 \mathrm{E}-3\left(\mathrm{~kg} \cdot \mathrm{m}^{2}\right)$, according to which the ranges of uncertain parameters in the dynamic equation are

$$
\begin{aligned}
& a_{1 \min }=1.0613 \leq a_{1}=B / J \leq 10.0700=a_{1 \max } \\
& b_{\min }=2.6484 \mathrm{E}+3 \leq b=K_{t} K_{v 2 c} / J \\
& \quad \leq 2.5128 \mathrm{E}+4=b_{\max }
\end{aligned}
$$

The nominal plant parameters in Eq. (1) are then assumed to be

$$
\left\{\begin{array}{l}
a_{1}=\left(a_{1 \max }+a_{1 \min }\right) / 2=5.5656 \\
b=\left(b_{\max }+b_{\min }\right) / 2=1.3888 \mathrm{E}+4, \quad d=0
\end{array}\right.
$$

in which no prior knowledge of external disturbance $\tilde{d}$ is assumed. When subject to a set-point change, the motor drive is desired to exhibit a non-overshooting response with a settling time of $0.25 \mathrm{sec}$. According to the proposed design procedure, we have

$$
\begin{aligned}
& \omega_{n}=7.5166 / 0.25=30.0664, \\
& K_{D}=\left(3 \omega_{n}-a_{1}\right) / b=0.0177 \\
& K_{P}=\left(3 \omega_{n}^{2}-a_{0}\right) / b=0.5660, \\
& K_{I}=\omega_{n}^{3} / b=5.6721 \\
& K_{0}=-3 / \omega_{n}=-0.0998
\end{aligned}
$$

As for the proposed disturbance observer, the switching gain is chosen as $\Psi=5.0$. In this system, the load is directly coupled to the shaft of the servomotor without any mechanical transmission. Hence, the main characteristic of the system is the uncertainties associated with load variations.

\subsection{Dynamic responses}

To test the effectiveness of the proposed controller, consider the following three cases of system inertia: $J \cong$ $8.79 \mathrm{E}-4\left(\mathrm{~kg} \cdot \mathrm{m}^{2}\right), J \cong 3.15 \mathrm{E}-3\left(\mathrm{~kg} \cdot \mathrm{m}^{2}\right)$, and $J \cong 3.32 \mathrm{E}-$ $4\left(\mathrm{~kg} \cdot \mathrm{m}^{2}\right)$ with an artificially introduced input disturbance

$$
\begin{aligned}
\tilde{d}= & -2.4 \sin (\pi(t-2.4) / 1.2) \cos (10 \pi(t-2.4)) \\
& \times[H(t-2.5)-H(t-3.5)]
\end{aligned}
$$

where $H(\cdot)$ denotes the unit step function. Figure 3 shows the rotor position responses for $J \cong 8.79 \mathrm{E}-4\left(\mathrm{~kg} \cdot \mathrm{m}^{2}\right)$ with the conventional and the proposed PID controllers, where the PID gains in the conventional PID controller, obtained by the pole-placement method, are of the same values as those in the proposed controller. It can be seen that the proposed PID controller fulfills the performance specifications and is superior to the conventional one in the sense that the proposed one suppresses overshoot of output responses, requires much smaller starting torque, and yields continuous control effort when the set point changes.

The performance of the proposed PID controller without perturbation compensation is presented in Fig. 4 with a consecutively changed step command. It can be seen that the output responses of the system are influenced to some extent by the change of load inertia. More obviously, the output responses are sensitive to input disturbances. Figure 5 shows the dynamic responses of the proposed PID-controlled system with a perturbation observer of Type I, in which $\omega_{0}=6 \omega_{n}$ in Eq. (37). It is clear that the output responses remain almost the same, irrespective of the variation of load inertia, demonstrating that the sensitivity to load variations has been greatly reduced by the perturbation compensation. Moreover, the amount of the 

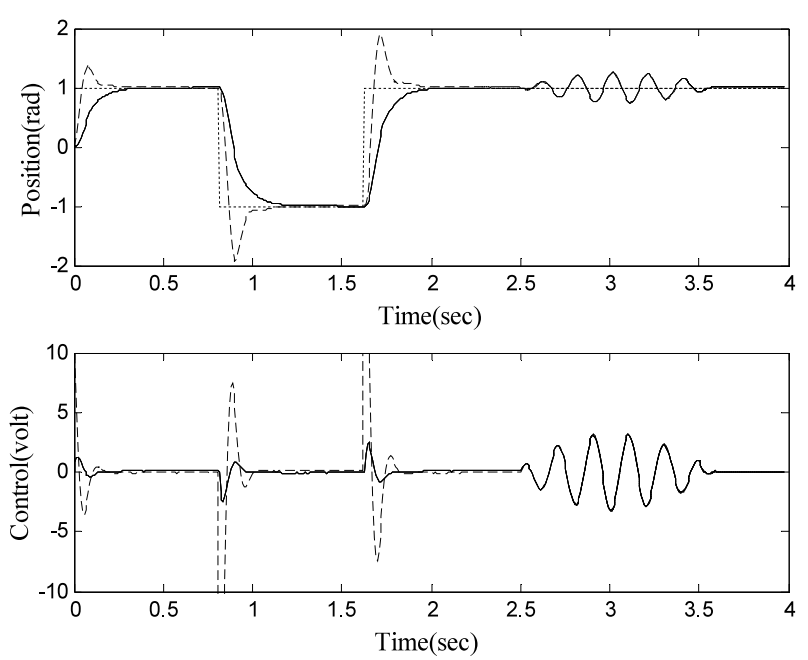

Fig. 3 Performance comparison between the proposed PID controller and the conventional one for $J \cong 8.79 \mathrm{E}-4(\mathrm{~kg}$. $\mathrm{m}^{2}$ ). Dotted line: reference command. Dashed line: the conventional PID controller. Solid line: the proposed PID controller
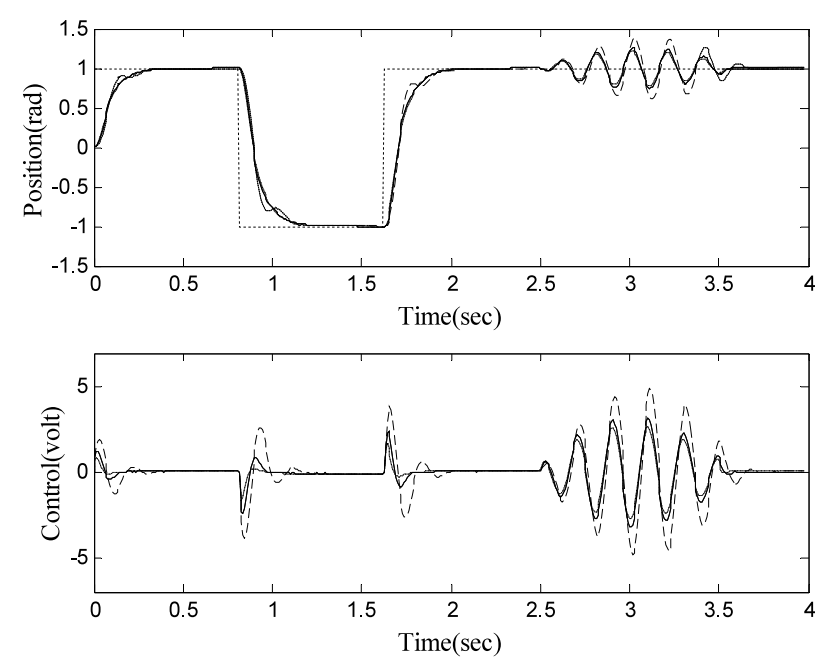

Fig. 4 Dynamic responses of the proposed PID control without perturbation compensation. Solid line for $J \cong 8.79 \mathrm{E}-$ $4\left(\mathrm{~kg} \cdot \mathrm{m}^{2}\right)$. Dashed line for $J \cong 3.15 \mathrm{E}-3\left(\mathrm{~kg} \cdot \mathrm{m}^{2}\right)$. Dashdot line for $J \cong 3.32 \mathrm{E}-4\left(\mathrm{~kg} \cdot \mathrm{m}^{2}\right)$. Dotted line for the reference command

maximum deviation from the desired position due to the input disturbance $\tilde{d}$ has been significantly decreased by the proposed perturbation compensation scheme without causing much chatter in the control signal. Regarding the Type-II perturbation observer, its bandwidth was adjusted to $\omega_{0}=10 \omega_{n}$ so that the level of chatter in the control is approximately equal to that for the Type-I perturbation observer with $\omega_{0}=6 \omega_{n}$. Figure 6 shows the dynamic responses with this Type-II perturbation observer. By comparing closely the output responses shown in Figs. 5 and 6 , we see that the maximum disturbance-induced position deviation with the Type-II compensation is about 0.84 times that with the Type-I compensation, illustrating that
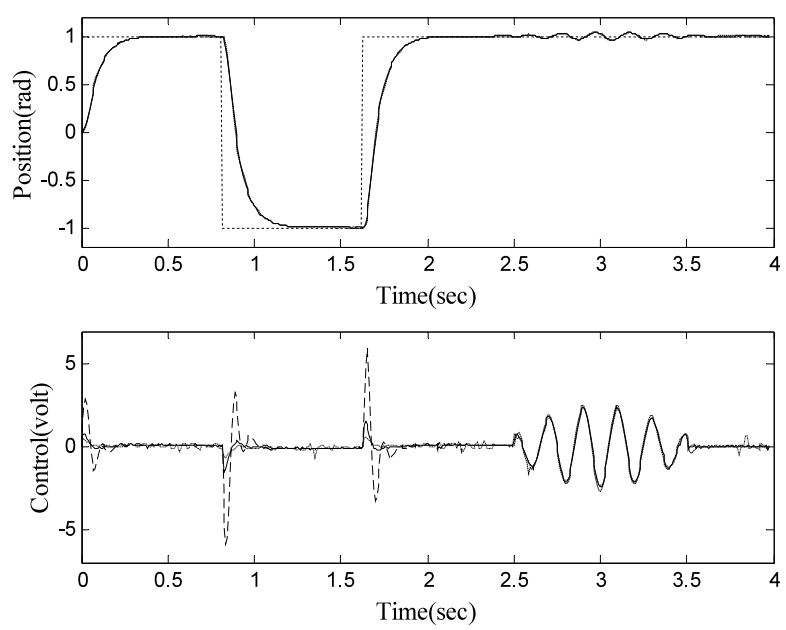

Fig. 5 Dynamic responses of the proposed PID control with a Type-I perturbation observer and $\omega_{0}=6 \omega_{n}$. Solid line for $J \cong 8.79 \mathrm{E}-4\left(\mathrm{~kg} \cdot \mathrm{m}^{2}\right)$. Dashed line for $J \cong$ $3.15 \mathrm{E}-3\left(\mathrm{~kg} \cdot \mathrm{m}^{2}\right)$. Dashdot line for $J \cong 3.32 \mathrm{E}-4\left(\mathrm{~kg} \cdot \mathrm{m}^{2}\right)$. Dotted line for the reference command
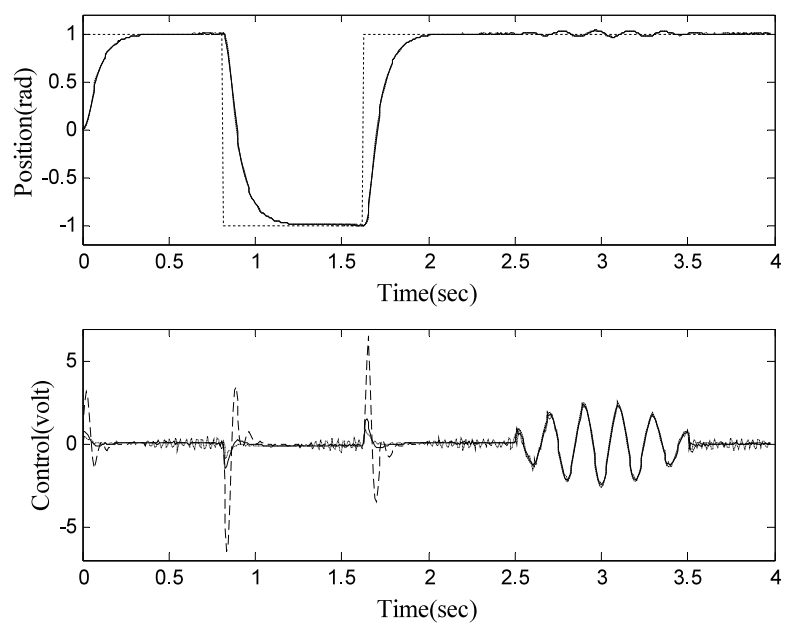

Fig. 6 Dynamic responses with a Type-II perturbation observer and $\omega_{0}=10 \omega_{n}$ tuned so that the chatter in the control has nearly the same level as that of the Type-I compensation with $\omega_{0}=6 \omega_{n}$. Solid line for $J \cong 8.79 \mathrm{E}-4\left(\mathrm{~kg} \cdot \mathrm{m}^{2}\right)$. Dashed line for $J \cong 3.15 \mathrm{E}-3\left(\mathrm{~kg} \cdot \mathrm{m}^{2}\right)$. Dashdot line for $J \cong 3.32 \mathrm{E}-4\left(\mathrm{~kg} \cdot \mathrm{m}^{2}\right)$. Dotted line for the reference command

the Type-II perturbation observer has better disturbancerejection capability than the Type-I observer with nearly the same chatter level in the control. However, the implementation of the Type-II perturbation observer demands more computation efforts than that of the Type-I observer. In sum, the system perturbation is well compensated for by the proposed perturbation observer, and the performance robustness of the PID-controlled system is much enhanced through perturbation compensation. The experimental results suggest that zero-overshooting responses can be achieved for step reference signals by the proposed scheme in spite of the presence of parameter uncertainties. 


\section{Conclusions}

For motor positioning systems, this paper has presented a non-overshooting PID controller with preassigned initial integral state and a perturbation compensation method. In the proposed PID control scheme, the PID gains are determined by the pole-placement method according to the desired settling time, and the initial integral state is so assigned that the nominal system exhibits non-overshooting output responses when the step reference command changes. The proposed PID controller can be designed by following a systematic design procedure and does not require the implementation of a reference model. Moreover, it yields continuous control effort and thus smooth system operations when subject to step changes in the reference signal.

To compensate for the system perturbation including the effects of parameter variations and unknown disturbances, the nominal design is augmented with a perturbation compensator that requires neither the accurate knowledge of system parameters nor access to the time derivative of the state vector. The dynamics of the PIDcontrolled system and that of the perturbation observer can be tuned separately, and varying the adaptation gain can adjust the performance of the perturbation compensation. In the proposed sliding perturbation observer, the switching gain needs only to be larger than the magnitude of the estimation error, instead of the whole unknown perturbation in the conventional design. This reduction of the required switching gain and the low-pass filtering characteristic of the integral perturbation estimation alleviate the chatter problem in the control, while the perturbation is precisely compensated for within the frequency range of interest. The combination of controller/observer yields a robust control structure for motor positioning systems. Experimental results confirm the effectiveness of the proposed scheme.

\section{Acknowledgements}

This work was supported by the National Science Council of ROC under grant number NSC 91-2622-E224-016-CC3.

\section{References}

( 1 ) Astrom, K.J. and Hagglund, T., Automatic Tuning of PID Controllers, (1988), ISA, New York.

( 2 ) Tan, K.K., Wang, Q.G., Hang, C.C. and Hagglund, T.J., Advances in PID Control, (1999), Springer-Verlag, London.

( 3 ) Cominos, P. and Munro, N., PID Controller: Recent Tuning Methods and Design to Specification, IEE Proc.-Control Theory Appl., Vol.149, No.1 (2002), pp.46-53.

(4) Ogata, K., Modern Control Engineering, (1997), Prentice-Hall, NJ.

( 5 ) Hang, C.C., Astrom, K.J. and Ho, W.K., Refinement of the Ziegler-Nichols Tuning Formula, IEE Proc.Control Theory Appl., Vol.138, No.2 (1991), pp.111118.

( 6 ) Astrom, K.J., Hang, C.C., Persson, P. and Ho, W.K., Towards Intelligent PID Control, Automatica, Vol.28, No.1 (1992), pp.1-9.

( 7 ) Eitelberg, E., A Regulating and Tracking PI(D) Controller, Int. J. Contr., Vol.45, No.1 (1987), pp.91-95.

( 8 ) Lin, F.J. and Liaw, C.M., Reference Model Selection and Adaptive Control for Induction Motor Drives, IEEE Trans. Automat. Contr., Vol.38, No.10 (1993), pp.1594-1600.

( 9 ) El-Sousy, F.F.M., Design and Implementation of 2DOF I-PD Controller for Indirect Field Orientation Control Induction Machine Drive System, Proc. of IEEE Int. Symposium Ind. Electr. (ISIE), Pusan, Korea, (2001), pp.1112-1118.

(10) Visioli, A., Tuning of PID Controllers with Fuzzy Logic, IEE Proc.-Control Theory Appl., Vol.148, No.1 (2001), pp.1-8.

(11) Visioli, A., Fuzzy Logic Based Set-Point Weighting Tuning of PID Controllers, IEEE Trans. Syst., Man, Cybern., Vol.29, No.6 (1999), pp.587-592.

(12) Lin, H.N. and Kuroe, Y., Decoupling Control of Robot Manipulators by Using Variable-Structure Disturbance Observer, Proc. of the 21st Intern. Conf. on Industrial Electronics, Control, and Instrumentation, (1995), pp.1266-1271.

(13) Chen, X., Komada, S. and Fukuda, T., Design of a Nonlinear Disturbance Observer, IEEE Trans. Ind. Electron., Vol.47, No.2 (2000), pp.429-437.

(14) Moura, J.T., Elmali, H. and Olgac, N., Sliding Mode Control with Sliding Perturbation Observer, ASME J. Dynamic Syst. Meas. Contr., Vol.119, No.4 (1997), pp.657-665.

(15) Lu, Y.S. and Chen, J.S., Design of a Perturbation Estimator Using the Theory of Variable-Structure Systems and Its Application to Magnetic Levitation Systems, IEEE Trans. Ind. Electron., Vol.42, No.3 (1995), pp.281-289.

(16) Utkin, V.I., Guldner, J. and Shi, J., Sliding Mode Control in Electromechanical Systems, (1999), Taylor \& Francis, London.

(17) Korondi, P., Young, K.D. and Hashimoto, H., Sliding Mode Based Disturbance Compensation for Motion Control, Proc. of the 23rd Intern. Conf. on Industrial Electronics, Control, and Instrumentation, (1997), pp.73-78.

(18) Young, K.D., Utkin, V.I. and Ozguner, U., A Control Engineer's Guide to Sliding Mode Control, IEEE Trans. Contr. Syst. Techn., Vol.7, No.3 (1999), pp.328342. 